\title{
Indoor Location Estimation Using Three Photodiodes and Ceiling LED Lights
}

\author{
Kanako Kinoshitaa,, , Shiyuan Yanga ${ }^{\mathrm{a}}$, Seiichi Serikawa ${ }^{\mathrm{a}}$ \\ ${ }^{a}$ Department of Electrical and Electronics Engineering, Kyushu Institute of Technology, Japan \\ *Corresponding Author: kinoshita.kanako408@mail.kyutech.jp
}

\begin{abstract}
In recent years, research on indoor autonomous mobile robots has been advanced. Accurate self-position estimation is necessary to enable autonomous movement. In this paper, we propose a method using LED lighting so that self-location can be estimated in various places.

The experiment used 3 photodiodes and 2 LED lights modulated. First, I modulate each of the two lights. Each illumination is received by three photodiodes attached to the robot's head, and the illuminance of each is obtained. The obtained values are fast Fourier transformed to distinguish between the two illuminations. Then, the radius is obtained from the illuminance and the position of the robot is estimated.

The conventional LED lighting method could only detect the position within the reach of one light. Moreover, since it could not be used in a place where light such as sunlight was mixed, it was lacking in practicality. However, this time, it was possible to estimate the position among multiple light sources by using modulated light and three photodiodes. In addition, since the device itself uses an inexpensive thing called a photodiode, it enables low-cost self-location estimation.
\end{abstract}

Keywords: photodiode, LED lighting, self-location.

\section{Introduction}

In recent years, unmanned transfer robots have been active in various places including automobile and factory production lines. At the present stage, it is mainly used in factories, but in the future it is thought that the field of robot activity will be further expanded. In order to make robots that can help people's lives, it is necessary to have a system that can move freely in various places instead of traveling only on a set course. And, in order to make it possible to move freely, it is necessary to have a technology to accurately recognize the robot's own location.

In this paper, we propose a system that recognizes indoor position using LED lighting as a marker so that it can be recognized in various places. In the conventional method, it could not be used in places where light such as sunlight are mixed. Moreover, although the information of the angle using a compass was also required, the accuracy of the compass was bad indoors and high-accuracy position estimation could not be performed. Therefore, in this case, three photodiodes can be used to cope with multiple lights, and accurate position measurement is possible.

\section{Principle of this research system}

\subsection{Overview of research system}

The image of this research system is shown in Fig. 2.1. The robot's head is equipped with three photodiodes, and LED lighting is used as a mark to estimate its own position. Moreover, we estimate self-location from information of two light sources so that multiple light sources can perform selflocation.

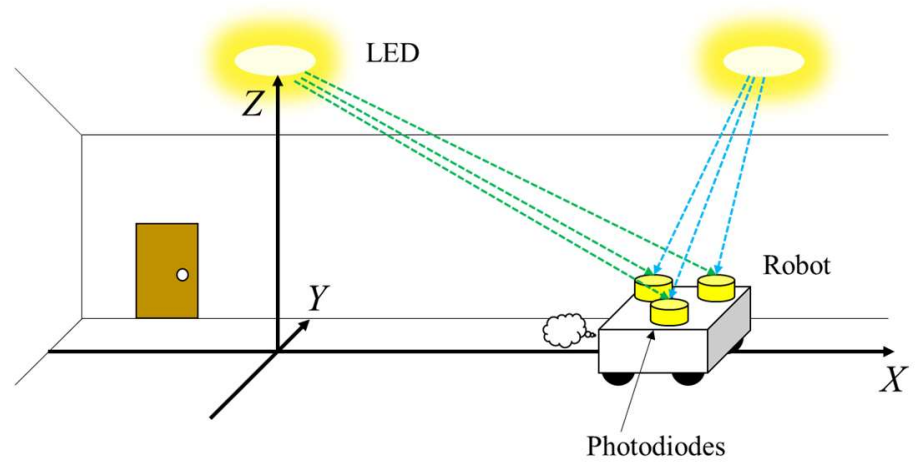

Fig. 2.1 System Image 


\subsection{Position detection by illuminance}

The cos 4 law is derived from the inverse square law of the distance and the oblique incident light characteristic regarding the illuminance. Then, the following equation is obtained.

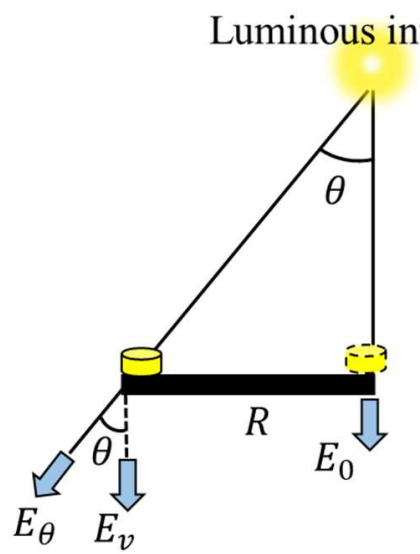

Fig. 2.2 Illumination distribution

$$
E_{v}=E_{\theta} \cos ^{4} \theta
$$

By using this equation, it is possible to predict the position by the illuminance.

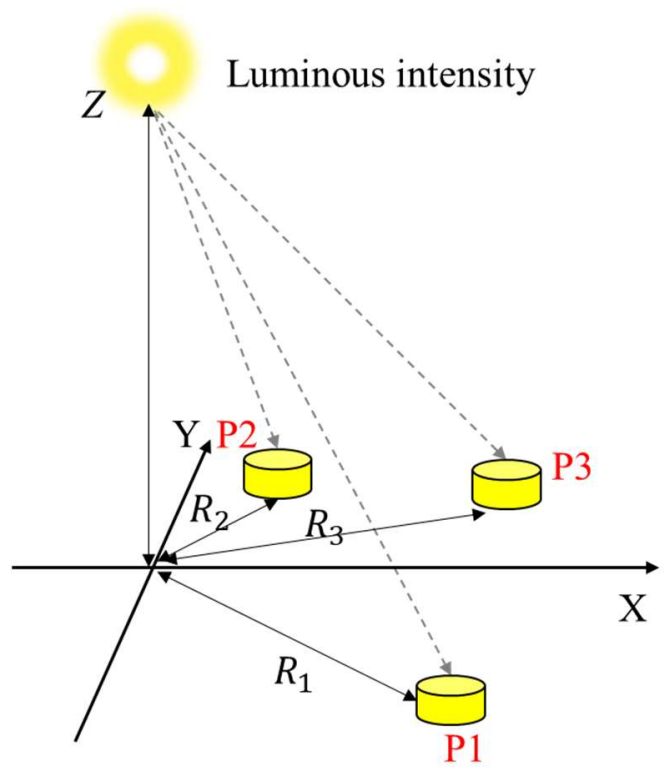

Fig. 2.3 Principle of detecting position from illuminance

Place three photodiodes arbitrarily as shown in Fig. 2.3. Also, let the origin be just below the light source. Let $Z$ be the distance from the light source to the origin, and $R_{1}, R_{2}$, and $R_{3}$ be the distances from the origin to the photodiodes $\mathrm{P} 1, \mathrm{P} 2$, and $\mathrm{P} 3$, respectively. The illuminance of P1, P2 and
P3 is as follows, assuming that the illuminance just below the light source is $E_{0}$ according to equation (2.1).

$$
E_{v, i}=E_{0} \cos ^{4} \theta \quad(i=1,2,3)
$$

The expression is rewritten as follows.

$$
\theta=\cos ^{-1}\left(\sqrt[4]{\frac{E_{v, i}}{E_{0}}}\right)
$$

Here, since $\theta$ is an angle formed by $P_{i}(i=1,2,3)$ directly below the light source and from the light source, it is expressed as follows.

$$
R_{i}=Z \tan \theta \quad(i=1,2,3)
$$

The equation (2.3) is substituted into the equation (2.4).

$$
R_{i}=Z \tan \left\{\cos ^{-1}\left(\sqrt[4]{\frac{E_{v, i}}{E_{0}}}\right)\right\}(i=1,2,3)
$$

Therefore, the distance between the photodiode and the origin can be obtained from the illuminance and the distance from the light source to the origin.

\subsection{Position detection with multiple light sources}

In general, when the light from multiple light sources is received simultaneously by the photodiode, it is necessary to light the light sources in order and to perform the synchronization process after the light is received by the photodiode. This is because when light from multiple light sources is received simultaneously, the output of the photodiode is a composite wave of the signals from the multiple light sources, making it impossible to distinguish each light source. In this section, we describe the asynchronous detection principle that enables simultaneous reception of multiple light sources with a photodiode.

First, modulated to different frequencies LED lighting to be used. When the light is received by the photodiode, the output signal of the photodiode is a composite wave of sine waves of different frequencies. By Fourier transforming the composite wave, an amplitude spectrum is obtained for each frequency. By reading the amplitude of the amplitude spectrum obtained at the modulation frequency of the LED illumination, it is possible to know the amount of light received by each LED illumination with the photodiode.

Here, the case where light of two LED illuminations is simultaneously received by a photodiode is described as an example below. Source of light $\mathrm{A}$ of bias voltage $A$, frequency $f 0$, amplitude $\alpha$ refers to bias voltage $B$, frequency $f 1$, a sine wave of source of light B of amplitude 
$\beta$ as follows each.

$$
\begin{aligned}
& v_{A}(t)=A+\alpha \sin 2 \pi f_{0} t \\
& v_{B}(t)=B+\beta \sin 2 \pi f_{1} t
\end{aligned}
$$

Upon receiving simultaneously a light source A and source $\mathrm{B}$ in the photodiode, the output signal of the photodiode is the sum of (2.6) and (2.7) below, are expressed as follows.

$$
v(t)=A+B+\alpha \sin 2 \pi f 0 t+\beta \sin 2 \pi f 1 t
$$

Using Euler's formula, equation (2.8) is expressed as follows.

$$
\begin{aligned}
v(t)= & A+B+\alpha 2 j(e j 2 \pi f 0 t-e-j 2 \pi f 0 t) \\
& +\beta 2 j(e j 2 \pi f 1 t-e-j 2 \pi f 1 t)
\end{aligned}
$$

(2.9) equation when Fourier transform is expressed as follows.

$$
\begin{gathered}
V(f)=2 \pi(A+B) \delta(f)+\alpha 2 j\{(\delta(f-f 0)-\delta(f+f 0)\} \\
+\beta 2 j\{(\delta(f-f 1)-\delta(f+f 1)\}
\end{gathered}
$$

The delta function is expressed as follows.

$$
\delta(f)=\{1(f=0) 0(f \neq 0)
$$

Therefore, the amplitude spectrum is expressed as follows.

$$
\begin{aligned}
& |V(f 0)|=\alpha 2 \\
& |V(f 1)|=\beta 2
\end{aligned}
$$

A spectrum is obtained at each modulation frequency by Fourier transforming a composite wave of time signals. Therefore, when received at the same time a photo light source A and source B modulated in different frequency diode, by Fourier transform of the composite wave, an amplitude spectrum for a light source A and source B is obtained at each modulation frequency.

\subsection{Position detection principle with photodiode and multiple light sources}

As shown in Fig. 2.4, the illumination is modulated to an arbitrary frequency, and map data in which the position and frequency of the LED illumination in the building are registered is given to the robot.

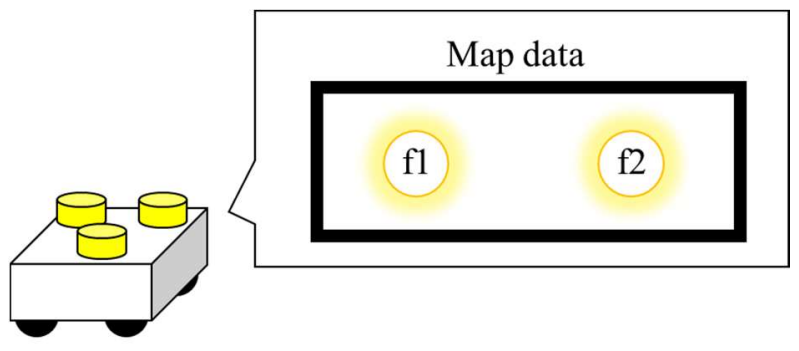

Robot

Fig. 2.4 Pre-mapping

The robot's photodiode output is read from the prediction of the change in illuminance for the position created as described above. As shown in Fig. 2.5, using the light reception information of one LED illumination, it is possible to specify the position of being on the circumference of a certain radius.

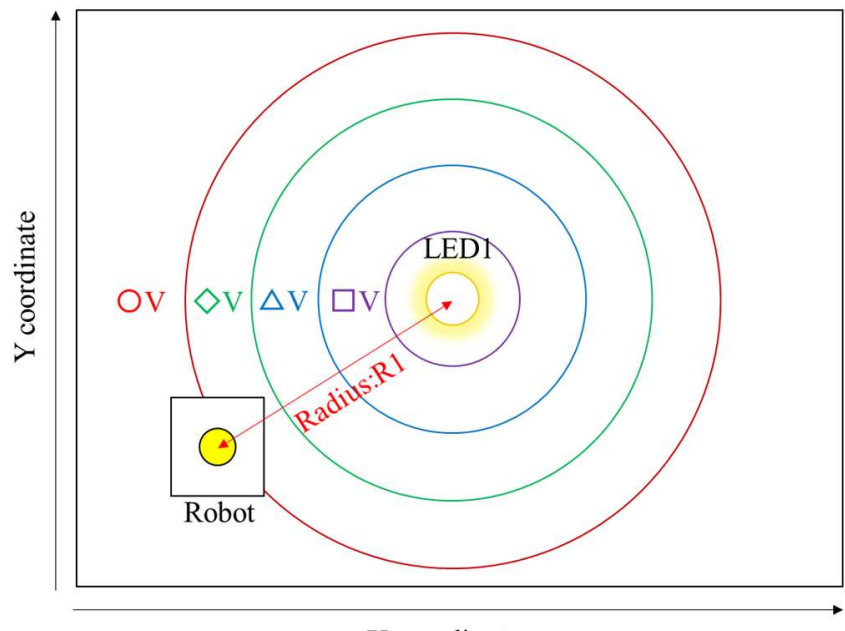

$\mathrm{X}$ coordinate

Fig. 2.5 Position detection using light reception information of one LED illumination

First, describing an expression specifying the position from the two information LED lighting as coordinates. With the center of one LED illumination as the origin as shown in Fig. 2.6, place another LED illumination on the $\mathrm{X}$ axis. And put the point $\mathrm{A}$ at any position.

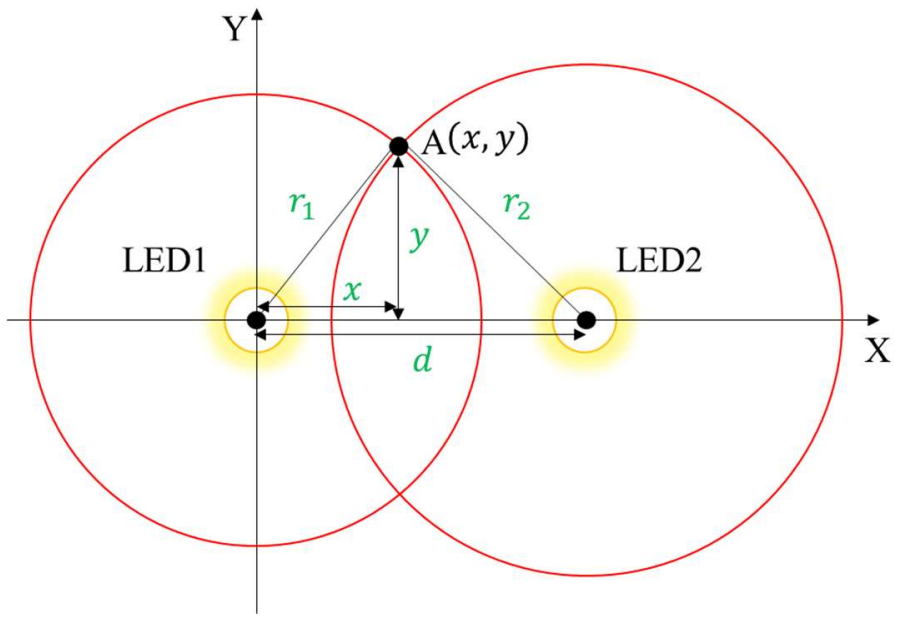

Fig. 2.6 Position detection in coordinates

Assuming that point $\mathrm{A}$ is $(x, y)$, two equations can be obtained as follows.

$$
\begin{gathered}
x^{2}+y^{2}=r_{1}{ }^{2} \\
(d-x)^{2}+y^{2}=r_{2}{ }^{2}
\end{gathered}
$$

When these equations are solved, it is expressed as follows. 


$$
\begin{gathered}
x=\frac{r_{1}{ }^{2}-r_{2}{ }^{2}+d^{2}}{2 d} \\
y=\sqrt{r_{1}{ }^{2}-\left(\frac{r_{1}{ }^{2}-r_{2}{ }^{2}+d^{2}}{2 d}\right)^{2}}
\end{gathered}
$$

However, it is not possible to pinpoint the position exactly as it is. As shown in Fig. 2.7, there are two types of position information. Three photodiodes are used to detect the position with two LED lights. Then, assuming the photodiodes A, B, and $\mathrm{C}$ as shown in Fig. 2.8, the position can be identified as one by judging the rotation direction.

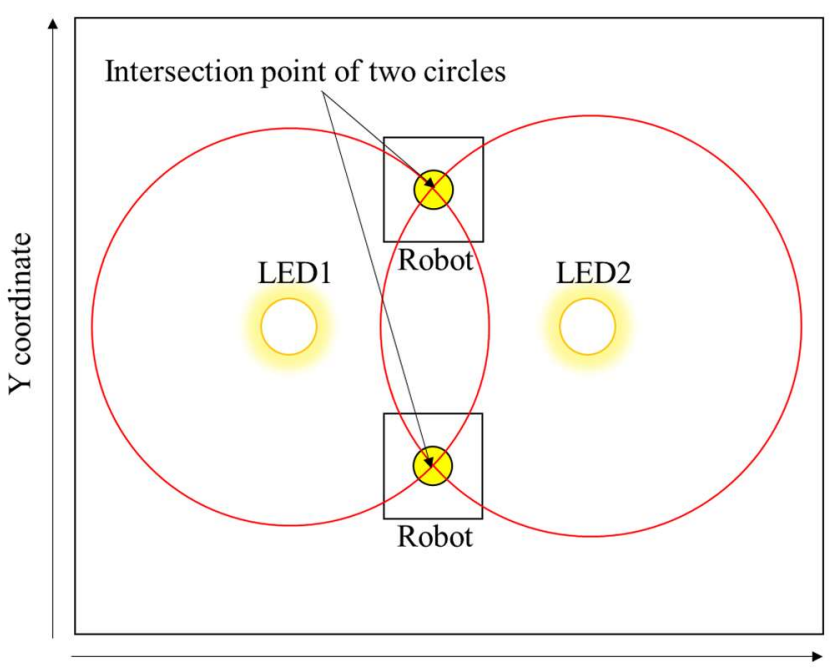

$\mathrm{X}$ coordinate

Fig. 2.7 Position detection using received light information of two LED lights

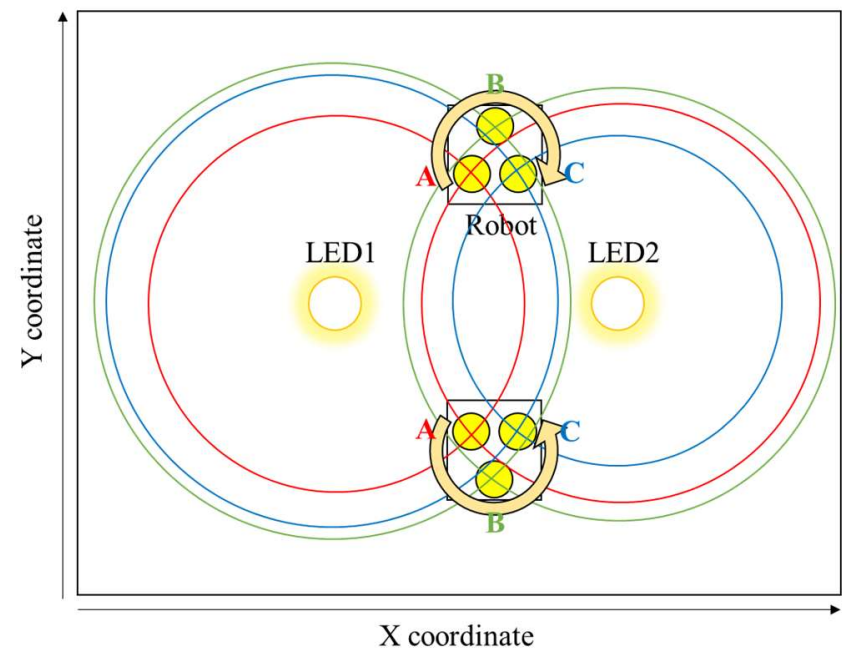

Fig. 2.8 Position measurement by rotation direction
The direction of rotation can be examined as follows. Let the center of rotation be point $\mathrm{A}$.

$$
\begin{aligned}
S= & \left(B_{x}-A_{x}\right) \cdot\left(C_{y}-A_{y}\right) \\
& -\left(B_{y}-A_{y}\right) \cdot\left(C_{x}-A_{x}\right)
\end{aligned}
$$

At this time, if $\mathrm{S}>0$, it is counterclockwise, and if $\mathrm{S}<0$, it is clockwise. If $\mathrm{S}=0$, then points $\mathrm{A}, \mathrm{B}$ and $\mathrm{C}$ are on a straight line.

\section{Experiment and result}

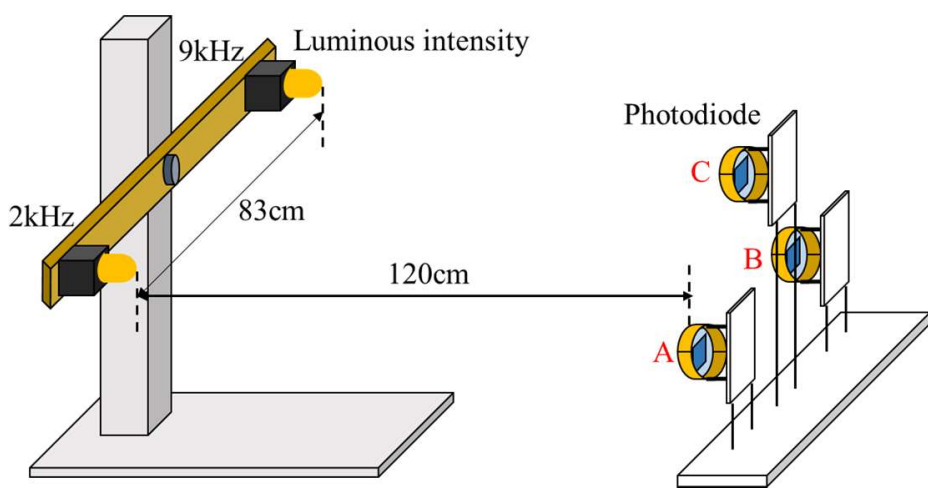

Fig. 3.1 Image of experiment

Place two LED lights and three photodiodes as shown in Fig. 3.1. The LED lighting is modulated to sine waves of $2 \mathrm{kHz}$ and $9 \mathrm{kHz}$ respectively.

\subsection{Experiment 1}

At first, using two LED lights and one photodiode, I inspected the precision with a real radius and the radius to be provided from an expression (2.5). Then the results shown in Table 3.1 were obtained.

Table 3.1 Results of Experiment 1

\begin{tabular}{|c|c|c|}
\hline $\begin{array}{c}\text { Theoretical value } \\
\text { (radius) }[\mathrm{cm}]\end{array}$ & $\begin{array}{c}\text { measured } \\
\text { value }[\mathrm{cm}]\end{array}$ & $\begin{array}{c}\text { Relative } \\
\text { error }\end{array}$ \\
\hline 20.00 & 21.60 & 0.08 \\
\hline 50.00 & 49.52 & 0.01 \\
\hline
\end{tabular}

From this result, the accuracy is less than $10 \%$, which is considered to be useful.

\subsection{Experiment 2}

Subsequently, the self-position was estimated from the information of three photodiodes. Then, the following results were obtained. Also, since the third photodiode $\mathrm{C}$ is used only after narrowing the position to two, photodiodes $\mathrm{A}$ and $\mathrm{B}$ are used for position detection. 
Table 3.2 Results of Experiment 2

\begin{tabular}{|c|c|c|c|c|}
\hline & \multicolumn{2}{|c|}{$\begin{array}{c}\text { Theoretical } \\
\text { value }\end{array}$} & \multicolumn{2}{c|}{ measured value } \\
\hline & $\mathrm{X}$ & $\mathrm{Y}$ & $\mathrm{X}$ & $\mathrm{Y}$ \\
\hline $\mathrm{A}$ & 20 & 0 & 21.60 & $\mathrm{NA}$ \\
\hline $\mathrm{B}$ & 50 & 0 & 49.52 & $\mathrm{NA}$ \\
\hline A & 30 & 0 & 30.48 & $\mathrm{NA}$ \\
\hline B & 60 & 0 & 60.53 & NA \\
\hline A & 20 & 10 & 21.79 & 7.67 \\
\hline B & 50 & 10 & 50.83 & 10.90 \\
\hline A & 30 & 10 & 30.94 & 9.99 \\
\hline B & 60 & 10 & 60.10 & 11.94 \\
\hline
\end{tabular}

Also, according to Table 3.2, when the $\mathrm{Y}$ axis value is 0 , an error occurs. The cause is considered to be that the inside of the square root becomes negative when using equation (2.17). Therefore, it is 0 when the system displays an error. A graph in which the error part is regarded as 0 is shown in Fig. 3.2.

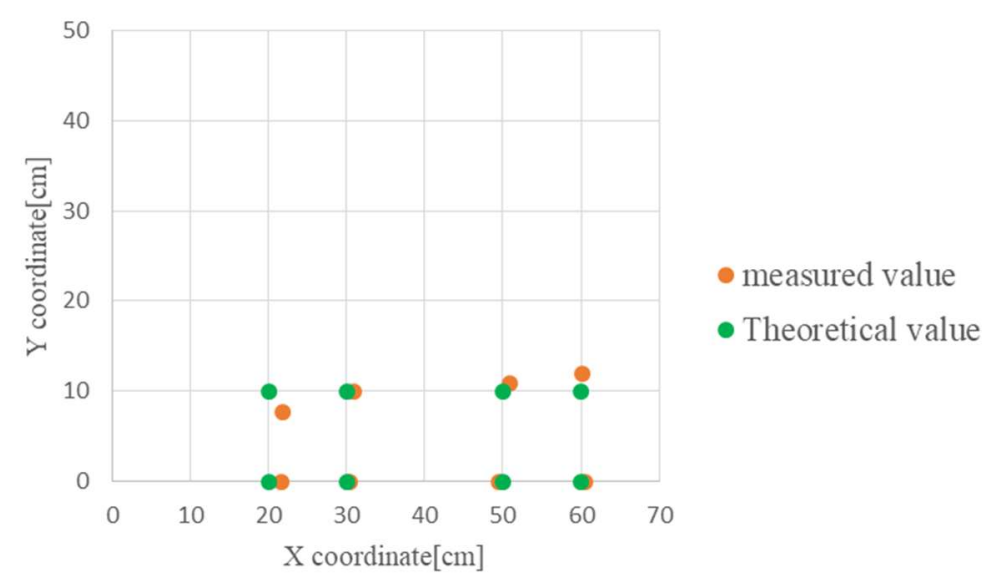

Fig. 3.2 Results of Experiment 2

\subsection{Consideration}

From the result of Experiment 1, it is considered that the accuracy of the radius is very high, and it is useful to use this in the equation. Also, from Experiment 2, the maximum error of the $\mathrm{X}$ coordinate is within $2 \mathrm{~cm}$, so the accuracy is considered very high. In addition, the maximum error of $Y$ coordinate is within $3 \mathrm{~cm}$, so it is considered useful. The cause of the error of the $\mathrm{Y}$ coordinate has become larger than the X-coordinate is believed that the equation (2.16) (2.17). When finding the $\mathrm{Y}$ coordinate, we used the $\mathrm{X}$ coordinate found by calculation. The error is included in this $\mathrm{X}$ coordinate itself, and it is thought that the error has increased because the square root is further calculated from there. In the future, by establishing a system that can detect the position with a simpler equation, it is considered that the position can be detected with higher accuracy.

\section{Conclusion}

By the method shown in this paper, we have established a system of high accuracy position detection. Moreover, by using the modulated LED illumination among multiple light sources, it was possible to extract only the necessary light information and incorporate it into the position detection system. Furthermore, since the maximum error as a whole could be suppressed within $3 \mathrm{~cm}$, it is considered that practical position detection could be performed. In the future, we would like to estimate the position using a simpler equation to improve the accuracy.

\section{References}

(1) Shiyuan Yang, Kenta Kagemoto, Kosuke Onishi: "Self-Position measurement for Indoor Using Ceiling LED Lights", Journal of the Institute of Industrial Applications Engineers, Vol.5, No.2, pp.86-89, 2017.

(2) Yoshino Masaki, Shinichiro Haruyama, Masao Nakagawa : "High-accuracy positioning system using visible LED lights and image sensor.”, 2008 IEEE Radio and Wireless Symposium. IEEE, pp.439-442, 2008.

(3) Nor Aida Mahiddin, Elissa Nadia Madi, Siti Dhalila, Engku Fadzli Hasan, Suhailan Safie and Noaizan Safie : "User Position Detection In An Indoor Environment.", International Journal of Multimedia and Ubiquitous Engineering, Vol.8, No.5, pp.303-312, 2013.

(4) Oe, Motoko, Tomokazu Sato, and Naokazu Yokoya :

"Estimating camera position and posture by using feature landmark database.”, Scandinavian Conference on Image Analysis. Springer Berlin Heidelberg, pp.171181, 2005. 